\title{
Effects of phenotypic complementarity and phylogeny on the nested structure of mutualistic networks
}

\author{
Enrico L. Rezende, Pedro Jordano and Jordi Bascompte \\ E. L. Rezende (enrico.rezende@ebd.csic.es), P. Jordano and J. Bascompte, Estación Biológica de Doñana, CSIC, Apartado 1056, \\ ES-41080 Sevilla, Spain.
}

\begin{abstract}
Recent studies have started to unravel the structure of mutualistic networks, although few functional explanations underlying such structure have been explored. We used computer simulations to test whether complementarity between phenotypic traits of plants and animals can explain the pervasive tendency of specialists to interact with proper subsets of species that generalists interact with (nested interactions), and how phylogeny affects such interaction patterns. Simultaneously, we assessed whether complementarity and phylogenetic structure were associated with patterns of interaction in a real mutualistic community. Simulation results support that highly nested networks can emerge from phenotypic complementarity, particularly when several traits are involved. The hierarchical structure of phylogenetic relations can also contribute to increased nestedness because traits determining complementarity are then inherited in a correlated fashion. Phylogenetic effects on resulting generalization levels are often low, but can be detected. Results from the empirical network support a relevant role of complementarity and phylogenetic history on interaction patterns. Our results demonstrate that these factors can contribute to nestedness, which emphasize the necessity of considering evolutionary mechanisms in studies of community structure.
\end{abstract}

Plant-animal mutualistic networks depict mutually beneficial interactions between plants and their animal pollinators or seed dispersers. An increasing number of studies currently attempt to understand how these mutualistic networks are structured and, more specifically, how predictable structures may emerge and/or evolve (Olesen and Jordano 2002, Bascompte et al. 2003, 2006, Jordano et al. 2003, 2006, Thompson 2005, Vázquez and Aizen 2006). A nested organization, in which specialists interact with proper subsets of the species generalists interact with, is an example of such a predictable structure (Bascompte et al. 2003, Bascompte and Jordano 2006). This pattern implies two properties at the network level. First, specialist species interact with generalists (Bascompte et al. 2003, Vázquez and Aizen 2004). Second, generalists are frequently associated among themselves building the 'core' of the network whereas 'peripheral' specialists tend to interact with this core of species (Bascompte et al. 2003, Vázquez et al. 2005). Properties such as nestedness probably have far reaching consequences on how coevolution proceeds in highly diversified and low-specificity mutualisms among free-living taxa (Thompson 2005, Lewinsohn et al. 2006).

Nestedness seems pervasive across mutualistic networks (Bascompte et al. 2003, Guimarães et al. 2006a, 2006b), whereas the processes underlying this network structure are not completely understood. Two candidates have been proposed as important factors underlying observed distributions of the number of links per species, and possibly nestedness: species abundance (Vázquez and Aizen 2004, Vázquez et al. 2007) and 'phenotypic complementarity' (Jordano 1987, Jordano et al. 2003, Stang et al. 2006). Phenotypic complementarity can be defined as the degree of functional matching between interacting species, and has been frequently gauged on key phenotypic traits that can directly affect the outcome of interactions (e.g. corolla length and tongue or bill length of pollinators, fruit size and gape width of animal frugivores, etc). Although the relevance of phenotypic complementarity as a determinant of species interactions is not new and has been 
acknowledged in studies addressing host shifts and specialized interactions (Nilsson 1988, Becerra 1997, Johnson and Steiner 1997, Borrell 2005, Nuismer and Thompson 2006) or community compartmentalization (Fonseca and Ganade 1996, Dicks et al. 2002, Prado and Lewinsohn 2004), its association with community nested structure has been so far a matter of speculation. For example, Thompson (2005) has suggested that nested patterns can result from the establishment of a core of highly interacting plants and animals via phenotypic complementarity and convergence within each set of species (see also Lewinsohn et al. 2006).

Several studies have described the relation between network structure of mutualistic communities and species abundance. Here, we determined whether phenotypic complementarity can be a plausible mechanism determining nested patterns of association, and under which conditions this may happen. For this purpose, we employed a simple model which assumes that phenotypic complementarity is the main determinant of how species interact and that generalization or specialization subsequently emerges from this process. Because related species tend to be phenotypically similar due to shared ancestry (Blomberg et al. 2003, Garland et al. 2005, Ives and Godfray 2006), we also address how phylogenetic relations can affect the outcome from our phenotypic complementarity model. We show that phenotypic complementarity results in increasingly higher levels of nestedness as the number of phenotypic traits involved increases, and that the hierarchical nature of species relationships can contribute significantly to the formation of nested patterns. In addition, we tested whether phenotypic complementarity and phylogenetic effects can account for interaction patterns of a real plant-seed disperser network.

\section{Material and methods}

\section{From phylogenies to interaction matrices}

At the community level, plant-animal mutualistic networks are often described by means of interaction matrices describing pairwise interactions among the two sets of plant (rows) and animal (columns) species, its graphical representation being a bipartite graph with the two sets of nodes connected by links (Jordano et al. 2003, 2006). In mutualistic networks, phylogenies depict hypotheses about the patterns of shared ancestry within each of the two sets of species (plants and animals) interacting in the network (Fig. 1). For our simulations, we chose 64 species for animals and 32 species for plants to maintain phylogenies completely balanced, and because these values fall within the range of mutualistic networks in nature (Bascompte et al.
2003, Jordano et al. 2003, Vázquez and Aizen 2004). We arbitrarily define 'animals' as the larger set of species and we will refer hereafter to plants and animals for clarity purposes, although we emphasize that these 'taxa' differ in the number of species used in our simulations but in no other attribute.

We employed Monte-Carlo simulations to simulate qualitative matrices of interactions ( 0 and 1 coding the absence and presence, respectively, of a pairwise interaction, Jordano et al. 2003) incorporating the effects of phylogenetic history for plants and animals. Simulations were performed in several steps, which are described in detail below. In summary, we first simulated the evolution of phenotypic traits of plant and animal species along their phylogenetic trees. Subsequently, we employed these phenotypic attributes to calculate the probability associated with the occurence of each animal-plant pairwise interaction (i.e. the probability that an animal species would interact with a plant species increases with the degree of matching between their phenotypes). These probabilities were then used to build the qualitative matrix of interactions. Therefore, the resulting network is determined solely by each species phenotypes and the phenotypes of their potential interactors. From these simulations, we subsequently analyzed (1) the structure of the qualitative matrix of interactions and (2) the number of interactions per species within the network (as mentioned above, generalization and specialization are emergent properties stemming from simulations).

We simulated the evolution of one to eight independent phenotypic traits for plants and animals assuming Brownian motion with PDSIMUL (Garland et al. 1993). We manipulated the relative contribution of phylogeny performing simulations across phylogenies with contrasting degrees of hierarchy (such contribution of phylogeny is currently known as 'phylogenetic signal', Blomberg et al. 2003). Succinctly, very hierarchical trees generally produce high phylogenetic signal (i.e. phylogenetically related species resembling each other) because a large proportion of the evolutionary history is shared between species. Conversely, in trees with little hierarchy most of the evolutionary history is independent between species (Fig. 1). Therefore, we initially simulated phenotypic evolution across star phylogenies, where phylogenetic signal should be absent because it lacks hierarchical structure (Fig. 1a). Subsequently, we repeated simulations employing phylogenies with Grafen's (1989) arbitrary branch lengths (Fig. 1b), which are considerably hierarchical and consequently should generate high levels of phylogenetic signal.

We then assigned each phenotypic trait in one species set (e.g. proboscis length of an insect) to a corresponding trait on the other set (e.g. the depth of a flower's corolla). Each pair of corresponding 
phenotypic traits is hereafter referred as a 'phenotypic dimension' (i.e. a pair of key traits in plants and animals whose matching can affect the outcome of the interaction). For example, the insect's nutrient requirements versus the flower's nectar and/or pollen composition, and activity period of an insect versus opening times of a flower, constitute two additional phenotypic dimensions. The probability of a plant-animal species pairwise interaction varies with the degree of 'matching' between their traits in each dimension, decreasing as a function of phenotypic distance between species. From two nonoverlapping distributions representing corresponding phenotypic traits for animals and plants, we calculated the probability $\mathrm{P}$ of interaction as a function of those traits as $1-d^{2}(a, p)$, where $d(a, p)$ is the standardized Euclidean distance (ranging from 0 to 1 ) between phenotypic traits of animal species a and plant species $p$ in a given dimension. Extrapolation to $\mathrm{n}$ phenotypic dimensions leads to:

$$
\mathrm{P}(\mathrm{a}, \mathrm{p})=\prod_{\mathrm{i}=1}^{\mathrm{n}}\left(1-\mathrm{d}_{\mathrm{i}}^{2}(\mathrm{a}, \mathrm{p})\right)
$$

To generate matrices with comparable connectances $(\mathrm{C}=$ the number of realized interactions/total possible interactions, Fig. 1) of $10 \%$ across simulations, probability values were then standardized so that their sum equals 0.1 .

Simulations were performed including 1, 2, 3, 4 and 8 different phenotypic dimensions for data with phylogenetic signal. For each permutation (i.e. with varying number of dimensions, with and without phylogenetic signal) we simulated 100 replicates, which represent 100 different sets of simulated traits in PDSIMUL. Interaction matrices were generated with $\mathrm{R}$ (Anonymous 2003), employing a module from the

Fig. 1. Schematic representation of mutualistic networks and the phylogenetic structure of plants and animals involved. Occupied cells depict a realized pairwise interaction. Each column and row depict a plant and an animal species, respectively, and animal and plant phylogenies are shown above and besides the interaction matrix. (a) Interaction matrix simulated from plant and animal phenotypic traits without phylogenetic signal (traits were simulated across a star phylogeny), with a connectance of $8.2 \%$ and nestedness $N=0.80$. (b) Matrix obtained from simulations of phenotypic traits with phylogenetic signal (phylogenies were balanced and highly hierarchical, resulting in high levels of signal), with a connectance of $7.9 \%$ and $\mathrm{N}=0.79$. Simulations in both cases were performed with 64 and 32 species of animals and plants, respectively, and 8 phenotypic dimensions (Fig. 2). (c) The plant-frugivore mutualistic network from the community of Nava de las Correhuelas, in Sierra de Cazorla, southeastern Spain, employed as an illustrative example in the text and Table 2 . This matrix contains 33 frugivore species and 25 plants, a connectance of $18.6 \%$ and $\mathrm{N}=0.93$.
PHYLOGR package (R. Diaz-Uriarte and T. Garland, available at http://cran.r-project.org) that reads output files from PDSIMUL and a program written ad hoc.
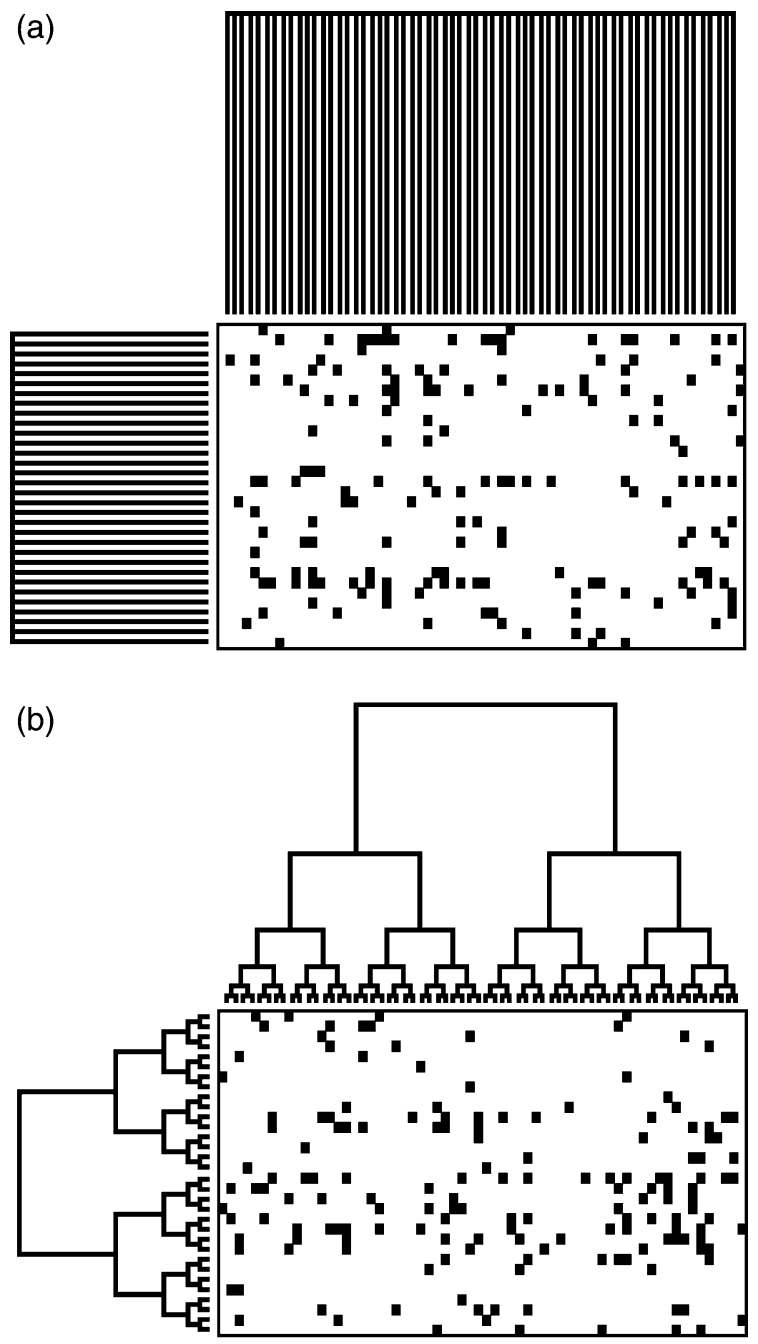

(c)

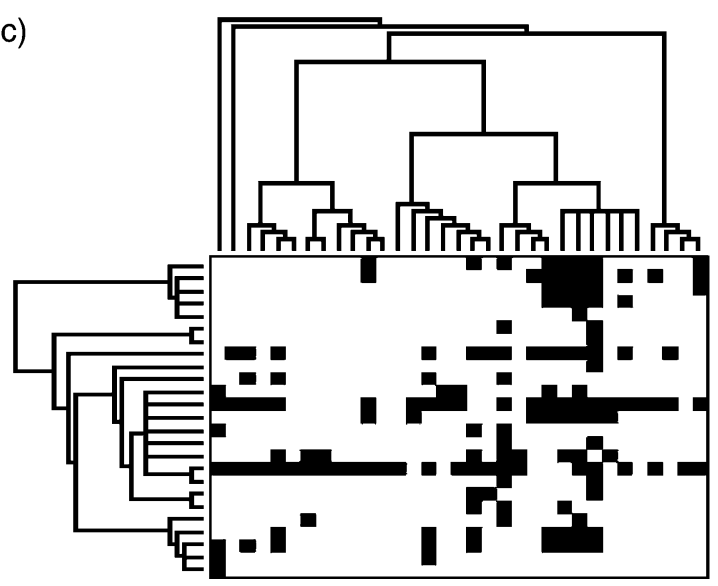




\section{Empirical dataset}

We assessed whether the association between patterns of interaction could be associated with phenotypic complementarity and phylogenetic history in a real interaction web. We studied the plant-frugivore network of the community of Nava de las Correhuelas, in Sierra de Cazorla, southern Spain (Fig. 1c), and tested whether interactions could be partly explained by the interplay between birds' beak size, and plants' fruit size and seed mass. Details about the study area and methods can be found in Jordano and Schupp (2000). Briefly, we carried out direct observations of frugivores feeding at fruiting plants and scored an interaction for each fruit handled by a frugivore (Snow and Snow 1988). These feeding records were taken either during timed watches at fruiting trees or during transect censuses (see Jordano 1993 for detailed methods). Observations were carried out yearly throughout the fruiting season between 1988-2000, with more sporadic observations between 2001-2005. A total of 7015 feeding records were obtained from $>520 \mathrm{~h}$ of observation.

Morphological measurements (bill length, width and height; $\pm 0.1 \mathrm{~mm}$ ) were performed on mist-netted birds and, for some species, from museum specimens (Estación Biológica de Doñana, CSIC, scientific collection). Fruit and seed size measurements (maximum length and width; $\pm 0.1 \mathrm{~mm}$ ) were performed on samples of 30 to 50 fruits from different individual plants (at least 5 distinct individuals, 6-10 fruits plant $^{-1}$ sampled). This data is available upon request to the authors.

Because our model assumes that the interaction between animal and plant phenotypic traits determine how species interact, we employed logistic and linear regressions to determine whether the interaction term is statistically associated with patterns of species assemblages. Significance of different factors in the logistic regressions were calculated from changes in maximum likelihood values, which follow a $\chi^{2}$ distribution with 1 DF. In addition, we tested whether morphological traits for plants and animals, as well the number of interaction per species in these groups, show significant phylogenetic signal, as explained below. Results are presented as mean $\pm S D$.

\section{Analyses}

We calculated the level of nestedness for each simulated matrix employing the 'temperature index' (Temp) of Atmar and Patterson (1993). This index estimates how an interaction matrix deviates on average from the isocline of perfect nestedness (i.e. how often unexpected interactions occur compared to a completely nested matrix), ranging from perfect nestedness $(\mathrm{Temp}=0)$ to perfect non-nestedness $($ Temp $=100)$. Temperatures were calculated employing Aninhado (Guimarães and Guimarães 2006, http://www.guimaraes.bio.br), which provides the same temperature outputs as the Nestedness Calculator software (Atmar and Patterson 1993) iteratively for large sets of matrices. Because here we emphasize nestedness or order instead of disorder, we calculated nestedness as $\mathrm{N}=(100-$ Temp $) / 100$ (Bascompte et al. 2003).

To test whether species propensity to interact and species realized number of interactions show significant phylogenetic signal (i.e. whether closely related species tend to significantly resemble each other), we employed the randomization procedure described by Blomberg et al. (2003). The underlying rationale is that, when species tend to resemble each other due to relatedness, the correlation between the phenotypic data and the structure of the candidate tree is high. If the correlation is higher than the correlation obtained for $95 \%$ of the randomizations where the phenotypic data has been randomly permuted across the tips of the tree (thus destroying any phylogenetic signal that may have existed), then one concludes that phylogenetically related species tend to resemble each other statistically more than expected at random (Blomberg et al. 2003). With the randomization procedure and the results from simulations with and without signal, we also quantified the statistical power to detect signal in our datasets ( 1 - type II error) and type I error rates, respectively (Blomberg et al. 2003).

To compare results from different simulations, the amount of phylogenetic signal was estimated with the $\mathrm{K}$ statistic proposed by Blomberg et al. (2003). The $\mathrm{K}$ statistic provides the fraction of signal of the data relative to the amount expected from Brownian motion (random walk) on a determined phylogeny. Thus, values of $\mathrm{K}$ equal to 1 indicate that the amount of resemblance between species is the same as the expectation from random walk along the candidate phylogeny. Values of K larger than 1 indicate that the data has more signal than expected for that tree, whereas values lower than 1 would indicate that there is less signal than expected (note that values of $\mathrm{K}$ can be low and remain statistically significant, depending on the results of the randomization procedure described above). For example, behavioral traits have $\mathrm{K}$ values falling consistently below 1, which suggests that behavior can evolve fast and result in more pronounced differences between closely related species than expected if behavior evolved following Brownian motion (Blomberg et al. 2003). We estimated $\mathrm{K}$ on both the arithmetic mean probability of each species to interact and on the realized number of interactions, to determine how the transformation from a continuous to a discrete variable might affect both the amount of signal and the statistical power to detect it (Blomberg et al. 2003). 
The effects of phylogenetic signal and number of phenotypic dimensions on nestedness were estimated with a nested ANCOVA. We compared simulations with and without signal (i.e. obtained employing a hierarchical and a star phylogeny, respectively; Fig. 1) and included the number of dimensions as a factor nested within these two groups. Not all species had an interaction and, therefore, did not participate in the interaction network. Thus, matrix size (i.e. the total number of possible interactions between animal and plant species $=$ spp. animals $\times$ spp. plants) and connectance (the number of realized interactions) were included as covariates.

\section{Results}

Simulations resulted in interaction matrices with variable sizes and connectances. Matrix size ranged between 1176 and 2048 cells (each cell represents a possible interaction) with an average size of $1773.8 \pm 130.2$ cells. Matrix mean connectance was $9.3 \pm 1.2 \%$, ranging between $6.7 \%$ to $18.6 \%$. Animal species included in the interaction networks varied between 42 and 64 species (mean of $56.9 \pm 3.2$ ), and plants included ranged between 25 and 32 species (mean $31.2 \pm 1.1$ ). Additional diagnostics (results not shown), such as the range of connectances obtained, the skewed distribution of links across species and the positive relationship between connectance and relative nestedness (Bascompte et al. 2003), support that our model generates matrices presumably comparable to real mutualistic communities.

Nestedness values across all simulations ranged between 0.64 and 0.90 (mean of $0.73 \pm 0.04$ ). All factors and covariates included in our nested analysis were statistically significant. The presence of phylogenetic signal and the number of phenotypic dimensions increased significantly nestedness on our ANCOVA $\left(\mathrm{F}_{1,988}=119.5, \quad \mathrm{p}<0.001\right.$ and $\mathrm{F}_{8,988}=79.1, \quad \mathrm{p}<$ 0.001 , respectively; Fig. 2). Adjusted means indicate that the increment from 1 to 8 phenotypic dimensions increased nestedness on average by $13.7 \%$ (Fig. 2). According to the coefficients obtained from the nested model, the presence of phylogenetic signal also contributed to increase nestedness by $3.3 \pm 0.4 \%$ (mean \pm $\mathrm{SE}$ ), and this effect was larger with additional number of dimensions (Fig. 2). Conversely, nestedness estimates were negatively correlated with matrix connectance $\left(\mathrm{F}_{1,988}=4.30, \mathrm{p}=0.038\right)$, perhaps because both variables vary with matrix size. Significant negative quadratic coefficients from regressions performed separately for datasets with and without signal $\left(\mathrm{t}_{497}=-4.2, \mathrm{p}<\right.$ 0.001 and $t_{497}=-2.9, p=0.003$, respectively) suggest a curvilinear association between nestedness and the number of dimensions employed (Fig. 2).
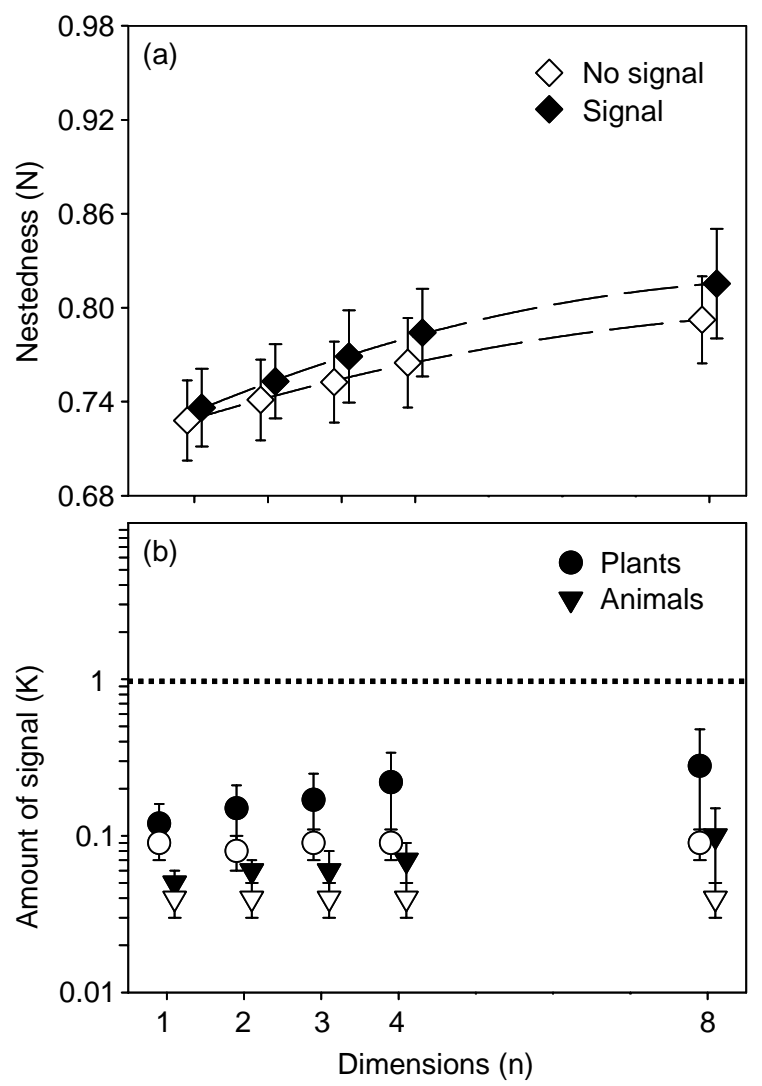

Fig. 2. (a) Nestedness and (b) amount of phylogenetic signal $\mathrm{K}$ of the number of links per species as a function of the number of dimensions ( $\mathrm{K}$ is the ratio of estimated signal over the expectation from the phylogeny assuming Brownian motion, hence $\mathrm{K}=1$ indicates a close match between phylogenetic relations among species and their phenotypic resemblance). Simulations were performed with and without phylogenetic signal (full and open symbols, respectively). Each point depicts means $\pm S D$ from 100 simulations, adjusted for a matrix with 57.7 animal species and 31.4 plant species and a connectance of $9.1 \%$. Dashed lines on (a) represent the following quadratic regressions: $\mathrm{N}=0.713+0.016$ $\mathrm{n}-0.0007 \mathrm{n}^{2}$ (without signal) and $\mathrm{N}=0.715+0.021 \mathrm{n}-$ $0.0011 \mathrm{n}^{2}$ (with signal).

Simulations without signal traced on a hierarchical phylogeny show that type I error rates fluctuate around $5 \%$, as expected (i.e. significant signal is detected when it is not present in $5 \%$ of the cases, Table 1). When phenotypic traits were simulated employing hierarchical trees, phylogenetic signal on the probability to interact per species (i.e. the average probability per row or column, see Fig. 1) decreased with the number of phenotypic dimensions added to the model, being significantly lower than the expectation from Brownian motion $(\mathrm{K}=1.0)$ in some instances (Table 1). The discretization from continuous probability values to the 
realized number of interactions per species caused an additional substantial decrease on the amount of phylogenetic signal (from a 2-fold to a 17-fold decrease). Paradoxically, both the amount of phylogenetic signal and the statistical power to detect signal (1-type II error) increased with the number of phenotypic dimensions included (Fig. 2, Table 1).

\section{Empirical network}

For the frugivory network of Nava de las Correhuelas, the interaction between beak size and seed mass is a weak but significant predictor of bird-plant interaction patterns. Results from backward and forward logistic regressions, employing measurements of beak height and width as surrogates for size, and fruit diameter and seed mass as plant characters (all log-transformed values), were always consistent. Main effects were never significant, whereas most bird versus plant interaction terms were statistically significant $\left(\chi^{2}=42.8, \mathrm{p}<\right.$ 0.001 for fruit diameter $\times$ beak width, $\chi^{2}=11.4$, $\mathrm{p}<0.001$ for seed mass $\times$ beak width and $\chi^{2}=23.9$, $\mathrm{p}<0.001$ for seed mass $\times$ beak height). Among realized pairwise interactions, a multivariate regression supported a significant positive correlation between beak height and seed mass $\left(n=130\right.$ interactions, $F_{1,129}=$ 3.07, 1-tailed $\mathrm{p}=0.041)$.

Regarding the phylogenetic component associated with patterns of interactions and morphological surrogates, randomization tests support significant phylogenetic signal on the number of links per species and morphological traits among birds, whereas in plants none of the variables showed significant phylogenetic signal (Table 2).

Table 2. Amount and significance of phylogenetic signal of variables from the community of Nava de las Correhuelas. $\mathrm{K}$ depicts the ratio of observed amount of resemblance between species in the phylogeny compared against expectations from a Brownian mode of phenotypic evolution. Analyses were performed employing constant branch lengths, as suggested by diagnostic tests. Values in bold indicate statistically significant phylogenetic signal $(p<0.05)$.

\begin{tabular}{|c|c|c|c|}
\hline & No. of species & K & $\mathrm{p}$ \\
\hline \multicolumn{4}{|l|}{ Animals } \\
\hline Beak widh & 28 & 1.340 & 0.001 \\
\hline Beak height & 28 & 1.766 & $<\mathbf{0 . 0 0 1}$ \\
\hline No. of links per sp. & 33 & 0.301 & 0.018 \\
\hline \multicolumn{4}{|l|}{ Plants } \\
\hline Fruit diameter & 25 & 0.308 & 0.173 \\
\hline Seed mass & 22 & 0.251 & 0.504 \\
\hline No. of links per sp. & 25 & 0.243 & 0.660 \\
\hline
\end{tabular}




\section{Discussion}

Few studies have integrated phylogenetic and complex network analyses (Cattin et al. 2004, Lewinsohn et al. 2005), and our study shows how integrating both areas can provide insights on unexplored mechanisms underlying community assemblages. Conversely, several studies have described the importance of species relative abundances for network patterns of interactions (Vázquez and Aizen 2006, Vázquez et al. 2007), or emphasized the role portrayed by network build-up processes as the determinants of species attributes in the network (Medan et al. 2007). Whereas these studies make no assumption about the intrinsic degree of specialization of species, our study assumes that phenotypic complementarity ultimately determines the number of interactors per species (see also Vázquez et al. 2005). Interestingly, both approaches can result in highly nested networks, hence interaction patterns in nature might ultimately result from complementary processes.

Nonetheless, observations such as the existence of syndromes (Fenster et al. 2004, Pauw 2006), or the absence of interaction between generalist species (Fig. 1c in Bascompte et al. 2003, Fig. 8.2 in Jordano et al. 2006), support that phenotypic complementarity probably contributes to community structure. In addition, the prevailing explanation based on abundance has been recently challenged, and neither sampling effort nor species abundance seem to totally account for species specialization levels (Blüthgen et al. 2006, Ghazoul 2006). In this context, although the role portrayed by abundance is certainly relevant, the enormous diversity of phenotypes encountered in nature and the potential consequences for interaction patterns should not be dismissed (Fontaine et al. 2006). For instance, the $16 \%$ increase in nestedness obtained as more dimensions and phylogenetic signal are included correspond to roughly $50 \%$ of the range of variation in nestedness observed in real mutualistic networks of similar size (Fig. 2 in Bascompte et al. 2003). In this context, new analytical methods may be able to partition the contribution of each factor to overall network structure, and also address how these factors interact. For instance, addressing whether species levels of generalization depend of their abundance or vice versa is a fundamental issue within this framework, with important repercussions on the evolution of these networks and on community stability.

Results listed here suggest that phenotypic diversity associated with evolutionary history can contribute to the structure of mutualistic networks. Instead of contradicting previous studies emphasizing the relevance of species abundance, we argue that these explanations are not mutually exclusive and should be integrated into a single more broad framework. For instance, it was recently suggested that nestedness and compartmentalization may result from different evolutionary and ecological processes, where 'compartmentation should reflect coevolutionary histories and constraints, whereas differences in species abundance or dispersal may generate nestedness' (Lewinsohn et al. 2006). Phenotypic complementarity has been invoked to explain the presence of compartments within networks of species interactions (Fonseca and Ganade 1996, Dicks et al. 2002, Jordano et al. 2003, 2006, Prado and Lewinsohn 2004, Wilson et al. 2004, Borrell 2005), and our results now suggest that it may partly account for nested patterns of mutualistic communities (Bascompte et al. 2003, Guimarães et al. 2006a, 2006b). Therefore, different patterns of association may be explained by similar processes, which opens the question of how the interplay between ecological and evolutionary processes generates such contrasting network structures (see also McGill et al. 2006).

\section{Phenotypic complementarity and network structure}

Our results show that the nested structure of mutualistic assemblages may be partly explained from differential patterns of phenotypic complementarity among species. Effects increase with the number of phenotypic dimensions because, as more dimensions may constrain possible interactions, the distribution of links are more heterogeneous and the effects of fewer generalist become increasingly important (Rezende unpubl). Although the existence of phenotypic preferences and/ or constraints associated with certain interactors seems intuitively obvious, testing which or how many phenotypic factors affect patterns of interactions in nature can be challenging in several ways (but see Ollerton and Liede 1997, Wilson et al. 2004, Borrell 2005, Muchhala 2006, Pauw 2006, Stang et al. 2006). First, the relationship between phenotypic values and interaction probabilities are difficult to quantify (with the exception of some forbidden interactions straightforward to identify, Jordano et al. 2006), and more so when one must consider phenotypic plasticity and/or individual variation. Second, the interaction between animal and plant traits may depend on additional factors (e.g. animal behavior). For instance, seed size might constrain seed dispersal for species that swallow the whole fruit, whereas other species which are pulp consumers may still disperse fruits with large seeds (Jordano 1995). Third, measurable phenotypic traits may be associated with several dimensions of 'complementarity' (e.g. body size alone could predict patterns of complementarity based on fruit size or nutritional quality if beak size and digestive efficiency vary as a function of size). Fourth, although some traits may be comparable across closely 
related species, comparisons between distant species may be practically impossible due to contrasting strategies underlying interactions (e.g. trying to compare pollination constraints associated with body size between bees and hummingbirds). Finally, some factors may be difficult to estimate, such as fruit or nectar palatability. These considerations highlight the challenges of studying the association between species phenotypes and community structure, and the necessity of a holistic approach in studies of this nature.

How can phenotypic complementarity be tested and quantified in natural systems? Studies with analogous models, where niche was defined as a hypervolume of $\mathrm{n}$ dimensions to study ecological niche space and overlap, have employed multivariate statistics (Litvak and Hansell 1990, see also McGill et al. 2006). However, the presence of two sets of species in plant-animal mutualistic systems provides an additional complication: interaction patterns may be determined by the interplay between phenotypes of animals and plants, besides the effects associated with phenotypic values of one of the sets as reported in some studies (Ackermann and Weigend 2006). Testing whether this is the case requires detailed information on phenotypic values of both sets of species, some a priori testable hypothesis of how phenotypic traits may determine patterns of interaction and interaction outcomes (Pauw 2006, Stang et al. 2006) and on species evolutionary history. For instance, beak size and gape width have been reported to influence and limit fruit foraging by avian frugivores (Wheelwright 1985, Levey 1987, Jordano 1995), and here we showed that the interaction between beak size and seed mass is a weak but significant predictor of bird-plant interaction patterns in the community of Nava de las Correhuelas. However, although general linear models may shed some light on the role portrayed by different traits and their interaction, this approach offers limited information on the causality of realized patterns. Experimental manipulation and preference trials may complement this strictly correlational approach, providing mechanistic explanations to observed patterns of interaction (e.g. the sugar composition of nectar and fruits partly determine the plant species with whom nectarivorous and frugivorous birds interact, Schondube and Martinez del Río, 2003).

\section{Phylogenetic signal}

Our findings suggest that, under certain scenarios, the presence of phylogenetic signal can significantly contribute to the nested structure of mutualistic networks, and this effect is larger as more phenotypic dimensions are involved (Fig. 2). How can phylogenetic structure result in more nested networks? It is known that, due to the hierarchical structure of species phylogenetic relations, closely related species will tend to be similar in several phenotypic traits because they inherit multiple traits from a single ancestor. For instance, two related bird species may have similar beak sizes, gut morphology and behave similarly (and so on). Therefore, distances across phenotypic dimensions will be correlated (even when their evolution is simulated separately, as in this study), and such correlation might explain why phylogenetically structured simulations are more nested. Two sources of evidence support this explanation. First, phenotypic distances were highly correlated across most dimensions in our simulations employing hierarchical phylogenies $(\mathrm{p} \ll 0.0001$, results not shown). Second, we were able to generate the same pattern observed in our simulations simply by varying the degree of correlation between phenotypic dimensions (Fig. 3). Notice that in this example we did not include any phylogenetic information. Instead, we sampled phenotypic values from different distributions that were more or less correlated between them.

Nevertheless, whether phylogenetic signal partly accounts for nested patterns of real communities remains to be tested, for several reasons. First, we currently do not know how many phenotypic dimensions might determine interaction patterns at a whole network level, even though relevant phenotypic constraints have been identified for some specific pairwise interactions (Stang et al. 2006). Second, the magnitude of phylogenetic signal can vary considerably in nature

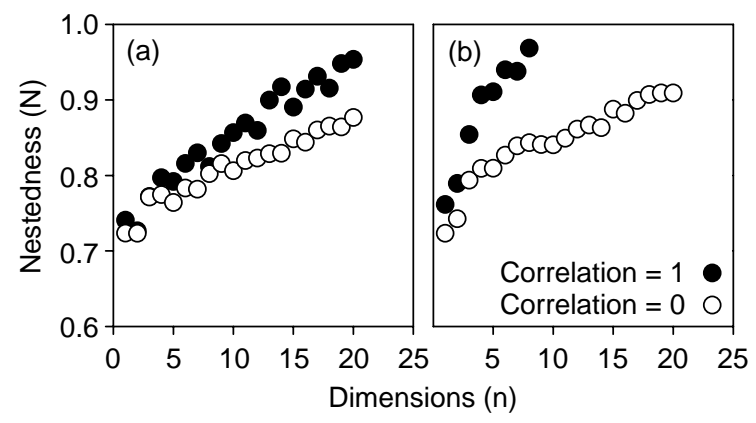

Fig. 3. Effects of the number of phenotypic dimensions $n$ on nestedness estimates for (a) the model depicted in main text (Eq. 1) and for (b) a model where the probabilities of interaction associated with each dimension are inversely proportional to phenotypic distances $\left(\sim 1 / d^{2}(a, p)\right)$. Simulations were performed assuming independent (open symbols) or highly correlated phenotypic dimensions (full symbols). In the later case, distances across dimensions are all identical, and probabilities vary in effect as a function of $1 /\left(d^{2}\right)^{n}$. Not all points are plotted in (b) because matrix size decreased dramatically when approaching 'perfect nestedness' $(\mathrm{N}=$ 1.0 ), as an increasingly larger fraction of species did not interact. 
depending on the phenotypic traits (see Blomberg et al. 2003 for a revision of $\mathrm{K}$ values on different sets of traits), and its effects on nestedness vary with the amount of signal. Accordingly, simulations with phylogenies with intermediate levels of signal resulted in nestedness values falling between the two curves shown in Fig. 2 (unpubl.). Third, different functions relating phenotypic distances with the probability of occurrence of a pairwise interaction provide qualitatively similar effects with varying magnitudes (Fig. 3). And fourth, as the number of dimensions increase, the network asymptotically approaches 'perfect nestedness' $(\mathrm{N}=$ 1.0 ), hence nestedness estimates obtained with and without phylogenetic signal should eventually converge.

Whether the number of links per species within a community presents significant phylogenetic signal, and how much signal is present, is currently not known (but see Rezende et al. 2007). The presence of significant signal on species propensity to interact may provide important information on the processes underlying network structure, and might reflect the existence of phenotypic constraints and/or preferences that determine interaction patterns, as well as differences in abundance that are associated with past evolutionary history (e.g. social versus asocial insects). In this context, our simulation results highlight two important caveats for future studies: (1) phylogenetic signal on the number of interactions per species can often be rescued from real datasets when interactions depend on traits with an important phylogenetic component, and (2) the amount of phylogenetic signal $\mathrm{K}$ will generally be low (Table 1), because the number of interactions is not continuous and because the propensity to interact is often determined by a set of phenotypic traits. Analyses performed on the mutualistic network of Nava de las Correhuelas (Fig. 1c) support the predictions listed above. Significant phylogenetic signal on the number of links per species among birds was coincident with signal on surrogates of beak size and of lower magnitude (Table 2), which was expected because morphological traits often show more phylogenetic signal that ecological traits. This pattern occurs because morphological traits tend to be more conserved along the phylogeny, and measurement errors tend to be lower in morphological measurements (Blomberg et al. 2003). Conversely, in plants none of the variables analyzed showed significant signal. These observations, taken together with the results from logistic regressions reported above, provides compelling evidence that phylogenetic effects on interaction patterns among animals may partly result from phenotypic complementarity. Nonetheless, more information is necessary to disentangle which factors underlie these results.

\section{Conclusions}

We have demonstrated that phenotypic complementarity and phylogenetic history can contribute significantly to nested patterns of interaction. Accordingly, recent studies have described the role of species phenotypes as determinants of interactions (Stang et al. 2006) and their relevance to ecosystem sustainability (Fontaine et al. 2006). It seems intuitively obvious that community structure should ultimately depend of species phenotypic attributes and abundances, and more general models including both factors are necessary. Such an integration is crucial to understand species and network tolerance to extinction events, specialization and generalization on ecological and evolutionary time, and the relevance of these processes on the evolution and stability of contrasting community structures.

Acknowledgements - This work was funded by the European Heads of Research Councils, the European Science Foundation, and the EC Sixth Framework Programme through a EURYI (European Young Investigator) Award (to JB), and by the Spanish Ministry of Education and Science (Grants REN2003-00273 and CGL2006-00373 to PJ and REN200304774 to JB). We are very grateful to P. R. Guimarães Jr. and D. P. Vázquez for their comments and insights on earlier versions of this study.

\section{References}

Ackermann, M. and Weigend, M. 2006. Nectar, floral morphology and pollination syndrome in Loasaceae subfam. Loasoideae (Cornales). - Ann. Bot. 98: 503514.

Anonymous 2003. R: a language and environment for statistical computing. - $\mathrm{R}$ Foundation for Statistical Computing, Vienna, Austria.

Atmar, W. and Patterson, B. D. 1993. The measure of order and disorder in the distribution of species in fragmented habitat. - Oecologia 96: 373-382.

Bascompte, J. and Jordano, P. 2006. The structure of plantanimal mutualistic networks. - In: Pascual, M. and Dunne, J. A. (eds), Ecological networks: linking structure to dynamics in food webs. Oxford Univ. Press, pp. 143159.

Bascompte, J. et al. 2003. The nested assembly of plantanimal mutualistic networks. - Proc. Natl Acad. Sci. 100: 9383-9387.

Bascompte, J. et al. 2006. Asymmetric coevolutionary networks facilitate biodiversity maintenance. - Science 312: $431-433$.

Becerra, J. X. 1997. Insects on plants: macroevolutionary chemical trends in host use. - Science 276: 253-256.

Blomberg, S. P. et al. 2003. Testing for phylogenetic signal in comparative data: behavioral traits are more labile. - Evolution 57: 717-745. 
Blüthgen, N. et al. 2006. Measuring specialization in species interaction networks. - BMC Ecol. 6: 9 (DOI 10.1186/ 1472-6785-6-9).

Borrell, B. J. 2005. Long tongues and loose niches: evolution of euglossine bees and their nectar flowers. - Biotropica 37: 664-669.

Cattin, M-.F. et al. 2004. Phylogenetic constraints and adaptation explain food-web structure. - Nature 427: $835-839$.

Dicks, L. V. et al. 2002. Compartmentalization in plantinsect flower visitor webs. - J. Anim. Ecol. 71: 32-43.

Felsenstein, J. 1985. Phylogenies and the comparative method. - Am. Nat. 125: 1-15.

Fenster, C. B. et al. 2004. Pollination syndromes and floral specialization. - Annu. Rev. Ecol. Evol. Syst. 35: 375403.

Fonseca, C. R. and Ganade, G. 1996. Asymmetries, compartments and null interactions in an Amazonian ant-plant community. - J. Anim. Ecol. 65: 339-347.

Fontaine, C. et al. 2006. Functional diversity of plantpollinator interaction webs enhances the persistence of plant communities. - PloS Biol. 4: 0129-0135.

Garland, T. et al. 1993. Phylogenetic analysis of covariance by computer simulation. - Syst. Biol. 42: 265-292.

Garland, T., Jr. et al. 2005. Phylogenetic approaches in comparative physiology. - J. Exp. Biol. 208: 3015-3035.

Ghazoul, J. 2006. Floral diversity and the facilitation of pollination. - J. Ecol. 94: 295-304.

Grafen, A. 1989. The phylogenetic regression. - Philos. Trans. R. Soc. Lond. B 326: 119-157.

Guimarães, P. R., Jr. and Guimarães, P. R. 2006. Improving the analyses of nestedness for large sets of matrices. - Environ. Modell. Soft. 21: 1512-1513.

Guimarães, P. R., Jr. et al. 2006a. Asymmetries in specialization in ant-plant mutualistic networks. - Proc. R. Soc. Lond. B 273: 2041-2047.

Guimarães, P. R., Jr. et al. 2006b. The nested structure of marine cleaning symbiosis: is it like flowers and bees? - Biol. Lett. 3: 51-54.

Ives, A. R. and Godfray, H. C. J. 2006. Phylogenetic analyses of trophic associations. - Am. Nat. 168: E1-E14.

Johnson, S. and Steiner, K. 1997. Long-tongued fly pollination and evolution of floral spur length in the Disa draconis complex (Orchidaceae). - Evolution 51: 45-53.

Jordano, P. 1987. Patterns of mutualistic interactions in pollination and seed dispersal: connectance, dependence asymmetries, and coevolution. - Am. Nat. 129: 657677.

Jordano, P. 1993. Geographical ecology and variation of plant-seed disperser interactions: southern Spanish junipers and frugivorous thrushes. - In: Fleming, T. and Estrada, A. (eds), Frugivory and seed dispersal: ecological and evolutionary aspects. Kluwer, pp. 85-104.

Jordano, P. 1995. Frugivore-mediated selection on fruit and seed size: birds and St. Lucies's cherry, Prunus mahaleb. - Ecology 76: 2627-2639.

Jordano, P. and Schupp, E. W. 2000. Seed disperser effectiveness: the quantity component and patterns of seed rain for Prunus mahaleb. - Ecol. Monogr. 70: 591615.
Jordano, P. et al. 2003. Invariant properties in coevolutionary networks of plant-animal interactions. - Ecol. Lett. 6: 69-81.

Jordano, P. et al. 2006. The ecological consequences of complex topology and nested structure in pollination webs. - In: Waser, N. M. and Ollerton, J. (eds), Specialization and generalization in plant-pollinator interactions. Univ. of Chicago Press, pp. 173-199.

Levey, D. 1987. Seed size and fruit-handling techniques of avian frugivores. - Am. Nat. 129: 471-485.

Lewinsohn, T. M. et al. 2005. Insects on plants: diversity of herbivore assemblages revisited. - Annu. Rev. Ecol. Syst. 36: 597-620.

Lewinsohn, T. M. et al. 2006. Structure in plant-animal interaction assemblages. - Oikos 113: 174-184.

Litvak, M. K. and Hansell, R. I. C 1990. A community perspective on the multidimensional niche. - J. Anim. Ecol. 59: 931-940.

McGill, B. J. et al. 2006. Rebuilding community ecology from functional traits. - Trends Ecol. Evol. 21: 178-185.

Medan, D. et al. 2007. Analysis and assembling of network structure in mutualistic systems. - J. Theor. Biol. 246: $510-521$.

Muchhala, N. 2006. The pollination biology of Burmeistera (Campanulaceae): specialization and syndromes. - Am. J. Bot. 93: 1081-1089.

Nilsson, L. 1988. The evolution of flowers with deep corolla tubes. - Nature 334: 147-149.

Nuismer, S. L. and Thompson, J. N. 2006. Coevolutionary alternation in antagonistic interactions. - Evolution 60: 2207-2217.

Olesen, J. M. and Jordano, P. 2002. Geographic patterns in plant-pollinator mutualistic networks. - Ecology 83: 2416-2424.

Ollerton, J. and Liede, S. 1997. Pollination systems in the Asclepiadaceae: a survey and preliminary analysis. - Biol. J. Linn. Soc. 62: 593-610.

Pauw, A. 2006. Floral syndromes accurately predict pollination by a specialized oil-collecting bee (Rediviva peringueyi, Melittidae) in a guild of Aouth African orchids (Coryciinae). - Am. J. Bot. 93: 917-926.

Prado, P. I. and Lewinsohn, T. M. 2004. Compartments in insect-plant associations and their consequences for community structure. - J. Anim. Ecol. 73: 1168-1178.

Rezende, E. L. et al. 2007. Non-random coextinctions in phylogenetically structured mutualistic networks. - Nature in press.

Schondube, J. E. and Martinez del Río, C. 2003. Concentration-dependent sugar preferences in nectar-feeding birds: mechanisms and consequences. - Funct. Ecol. 17: 445453.

Snow, B. K. and Snow, D. W. 1988. Birds and berries. - T. and A. D. Poyser, Calton, UK.

Stang, M. et al. 2006. Size constraints and flower abundance determine the number of interactions in a plant-flower visitor web. - Oikos 112: 111-121.

Thompson, J. N. 2005. The geographic mosaic of coevolution. - Univ. of Chicago Press.

Vázquez, D. P. and Aizen, M. A. 2004. Asymmetric specialization: a pervasive feature of plant-pollinator interactions. - Ecology 85: 1251-1257. 
Vázquez, D. P. and Aizen, M. A. 2006. Community-wide patterns of specialization in plant-pollinator interactions revealed by null models. - In: Waser, N. M. and Ollerton, J. (eds), Plant-pollinator interactions: from specialization to generalization. Univ. of Chicago Press, pp. 200-219.

Vázquez, D. P. et al. 2005. Species abundance and the distribution of specialization in host-parasite interaction networks. - J. Anim. Ecol. 74: 946-955.
Vázquez, D. P. et al. 2007. Species abundance and asymmetric interaction strength in ecological networks. - Oikos in press.

Wheelwright, N. 1985. Fruit size, gape width, and the diets of fruit-eating birds. - Ecology 66: 808-818.

Wilson, P. et al. 2004. A multivariate search for pollination syndromes among penstemons. - Oikos 104: 345361. 